\title{
Deletion of M-Opsin Prevents M Cone Degeneration in a Mouse Model of Leber Congenital Amaurosis
}

Hui Xu, ${ }^{* \dagger}$ Nduka Enemchukwu, ${ }^{\dagger \ddagger}$ Xiaoyue Zhong, ${ }^{\dagger \dagger \S}$ Olivia Zhang, ${ }^{\dagger \dagger \S}$ and Yingbin Fu*

From the Interdepartmental Program in Neuroscience, * Department of Ophthalmology, University of Utah, Salt Lake City, Utah; Departments of Ophthalmology ${ }^{\dagger}$ and Neuroscience, ${ }^{\ddagger}$ Baylor College of Medicine, Houston, Texas; and Rice University, ${ }^{\S}$ Houston, Texas

\author{
Accepted for publication \\ January 7, 2020. \\ Address correspondence to \\ Yingbin Fu, Ph.D., Department \\ of Ophthalmology, Baylor \\ College of Medicine, 6550 \\ Fannin St., Neurosensory Bldg, \\ NC 506, Houston, TX \\ 77030. E-mail: yingbin.fu@ \\ bcm.edu.
}

\begin{abstract}
Mutations in retinoid isomerase (RPE65) or lecithin-retinol acyltransferase (LRAT) disrupt 11-cis-retinal synthesis and cause Leber congenital amaurosis (LCA). Despite the success of recent RPE65 gene therapy, follow-up studies show that patients continue to experience photoreceptor degeneration and lose vision benefit over time. In $\mathrm{Lrat}^{-/-}$mouse model, mislocalized medium (M)-wavelength opsin was degraded, whereas mislocalized short $(S)$-wavelength opsin accumulated before the onset of cone degeneration. The mechanism for the foveal M/long-wavelength cone photoreceptor degeneration in LCA is unknown. By crossing $\mathrm{Lrat}^{-/-}$mice with a proteasome reporter mouse strain, this study showed that M-opsin-enriched dorsal cones in $\mathrm{Lrat}^{-/}$mice exhibit proteasome stress because of the degradation of large amounts of $M$-opsin. Deletion of $M$-opsin relieves the proteasome stress and completely prevents $\mathrm{M}$ cone degeneration in $\mathrm{Lrat}^{-/-} \mathrm{Opn} 1 \mathrm{sw} \mathrm{C}^{-/-}$mice (a pure M cone LCA model, Opn1sw encoding S-opsin) for at least 12 months. These results suggest that M-opsin degradation-associated proteasome stress plays a major role in $\mathrm{M}$ cone degeneration in $\mathrm{Lrat}^{-/-}$model. This finding may represent a general mechanism for $M$ cone degeneration in multiple forms of cone degeneration because of $M$-opsin mislocalization and degradation. These results have important implications for the current gene therapy strategy for LCA that emphasizes the need for combinatorial therapies to both improve vision and slow photoreceptor degeneration. (Am J Pathol 2020, 190: 1059-1067; https://doi.org/10.1016/ j.ajpath.2020.01.005)
\end{abstract}

Retinoid isomerase (RPE65) and lecithin-retinol acyltransferase (LRAT) are two key enzymes involved in the generation/recycling of 11-cis-retinal in the retinal pigment epithelium (RPE). Mutations in either gene lead to Leber congenital amaurosis (LCA), a severe inherited retinal degenerative disease characterized by severe loss of vision in childhood with early degeneration of foveal cones followed by rods. ${ }^{1,2}$ Two mouse models, Rpe65 ${ }^{-1-}$ (LCA2) and $\mathrm{Lrat}^{-1-}$ (LCA14) mice, which have shown similar phenotypes, and an Rpe65 $5^{-1-}$ dog model have been widely used for mechanistic and therapeutic studies. ${ }^{3-11}$ These studies paved the way for the first successfully treated inherited retinopathy using gene therapy. ${ }^{12-14}$ However, follow-up studies found that photoreceptor degeneration continued even after the gene therapy intervention in LCA $2,{ }^{11,15-17}$ suggesting a need to understand the mechanism of photoreceptor degeneration to design improved treatment strategies.

In both LCA patients and animal models, both rod and cone functions are severely compromised because of a combination of 11-cis-retinal deficiency and photoreceptor degeneration. Early loss of foveal cones was reported in RPE65-deficient patients. ${ }^{1,2}$ Despite extensive studies, the mechanisms underlying cone degeneration in

Supported by NIH grants EY022614 (Y.F.), 2P30EY002520 (Baylor College of Medicine), and 5P30EY014800 (University of Utah); a Retina Research Foundation grant (Y.F.); the Sarah Campbell Blaffer Endowment in Ophthalmology (Y.F.); and Research to Prevent Blindness unrestricted grants (Department of Ophthalmology, University of Utah, and Department of Ophthalmology, Baylor College of Medicine).

Disclosure: None declared. 

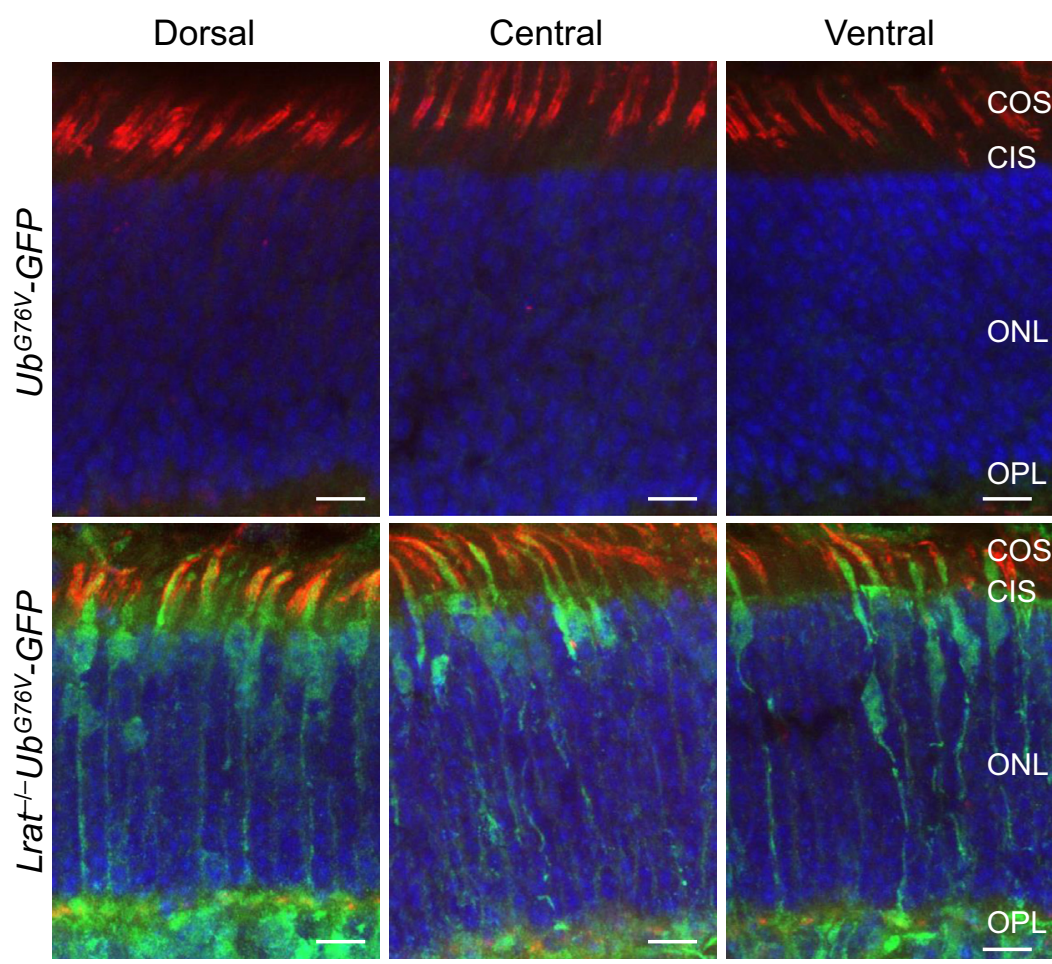

Figure 1 Cones in lecithin-retinol acyltransferase deficient $\left(\mathrm{Lrat}^{-/-}\right)$mice exhibit proteasome insufficiency. The retinal sections from P18 $\mathrm{Lrat}^{-/-}$UbG76V-GFP and UbG76V-GFP control mice were labeled with an anti-green fluorescent protein (GFP) antibody (green). Cones were labeled with rhodamine-peanut agglutinin (red). Nuclei were stained with DAPI. Scale bars $=10 \mu \mathrm{m}$. CIS, cone inner segment; COS, cone outer segment; ONL, outer nuclear layer; $\mathrm{OPL}$, outer plexiform layer.
LCA are still poorly understood. In mouse models, both medium-wavelength sensitive opsin (M-opsin) and shortwavelength sensitive opsin (S-opsin) fail to traffic from the cone inner segment to the outer segment and cone photoreceptors in the central/ventral retina degenerate rapidly ( $<4$ weeks). ${ }^{5-7,18}$ Dorsal cones degenerate slowly ( $>6$ months). It has been shown previously that $\mathrm{S}$-opsin was aggregation prone in cell culture and accumulated in cones, leading to proapoptotic endoplasmic reticulum (ER) stress and rapid S-opsin enriched central/ventral cone (S-cone) degeneration in Lrat $^{-1-}$ mice. ${ }^{8}$ Deletion of S-opsin reduced ER stress and completely prevented the rapid $\mathrm{S}$-cone degeneration in $\mathrm{Lrat}^{-1-} \mathrm{S}$-opsin ${ }^{-1-}$ mice for at least 12 months. ${ }^{19}$ However, the mechanism for $\mathrm{M}$ opsin enriched dorsal cone ( $\mathrm{M}$ cone) degeneration is unclear. Understanding the mechanism for $\mathrm{M} / \mathrm{L}$ (medium/ long-wavelength) cone degeneration is important because the human fovea is dominated by both cone types, which are responsible for high-resolution daylight vision and color perception. ${ }^{20}$

Previous work has shown that mistargeted M-opsin was largely degraded, whereas S-opsin was resistant to degradation in $\mathrm{Lrat}^{-1-}$ mice. ${ }^{8} \mathrm{M}$-opsin was shown to be degraded by the proteasome but not lysosome pathway in Rpe $65^{-1-}$ mice. $^{21}$ Degradation of large amounts of mistargeted Mopsin in cones will likely overload proteasome and cause proteasome stress. Interference with the proteasome inhibits both cytosolic protein degradation and ER-associated degradation, which can lead to cell death. Indeed, the degradation of rod transduction proteins was shown to cause proteasome overload, ER stress, and rod degeneration. ${ }^{22-24}$
Moreover, decreased proteasomal activity causes rod degeneration, ${ }^{25}$ whereas increased proteasomal activity delays rod degeneration. ${ }^{26}$ However, the role of proteasome stress in cone degeneration has not been studied. Because of mixed expression of $\mathrm{M}$-opsin and $\mathrm{S}$-opsin in mouse cones and the rapid degeneration caused by $\mathrm{S}$-opsin in $\mathrm{Lrat}^{-1-}$

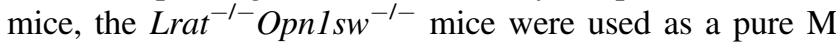
cone LCA model to study the role of M-opsin and proteasome stress in cone degeneration. This study showed that deletion of $\mathrm{M}$-opsin relieves the proteasome stress and prevents $\mathrm{M}$ cone degeneration in $\mathrm{Lrat}^{-/-} \mathrm{OpnIsw}^{-/-}$mice for at least 12 months.

\section{Materials and Methods}

\section{Mice}

M-opsin ${ }^{-1-}$ mice were generated by clustered regularly interspaced short palindromic repeats and CRISPR-associated protein 9 (CRISPR-Cas9) following a published protocol. ${ }^{27}$ Single-guide RNA (5'-ATTATCACATTGCTCCCAGG-3') was designed to target the second exon of mouse $\mathrm{M}$-opsin gene Opn1mw. Cas9 plasmid and single-guide RNA were coinjected into $\mathrm{C} 57 \mathrm{Bl} / 6 \mathrm{~J}$ mouse embryos at the University of Utah Transgenic/Gene Targeting Core Facility (Salt Lake City, UT). Mouse tail samples from Opn $1 \mathrm{mw}^{-1-}$ founder mice were prepared and used as PCR template with forward -primer (5'-CATAGAGCAAGGAAAAGTGAGGTC- $3^{\prime}$ ) and reverse primer (5'-CCCAGAACGAAGTAGCCATAGAT- $\left.3^{\prime}\right)$. The PCR product was gel purified and sequenced to select the 
founder mice. The pups of the Opn $1 m w^{-1-}$ founder mice were confirmed by DNA sequencing. $\mathrm{Lrat}^{-1-}, \mathrm{Opnlsw}^{-/-}$, and $\mathrm{Lrat}^{-1-}$ Opn1 $\mathrm{sw}^{-1-}$ mice were generated previously. ${ }^{4,19,28}$ Opn1sw ${ }^{-1-}$ Opn $1 m w^{-1-}$ mice were produced by crossing the Opn1sw ${ }^{-1-}$ and Opn1mw ${ }^{-1-}$ strains. Lrat $^{-1-}$ Opnlsw $^{-1-}$ $O p n 1 m w^{-1-}$ mice were produced by crossing the $\mathrm{Lrat}^{-1-}$ S-opsin ${ }^{-1-}$ and M-opsin ${ }^{-1-}$ strains. Lrat ${ }^{-1-}$ Opn1sw ${ }^{-{ }_{-}} \mathrm{Ub}^{\mathrm{G} 76 \mathrm{~V}}{ }_{-}$GFP and $\mathrm{Lrat}^{-1-} \mathrm{Opn1} \mathrm{sw}^{-1-}$ OpnImw ${ }^{-1-} \mathrm{Ub}^{\mathrm{G} 76 \mathrm{~V}}$ GFP mice were produced by crossing the $\mathrm{Lrat}^{-1-} \mathrm{Opnl}_{\mathrm{sw}}{ }^{-1-}$ and $\mathrm{Lrat}^{-1-}$ OpnIsw ${ }^{-1-}$ OpnImw ${ }^{-1-}$ strains, respectively, with the reporter $U b^{G 76 V}-G F P$ mice. Mice were reared under cyclic light (12 hours light/12 hours dark). Animal experiments were approved by the Institutional Animal Care and Use Committees at the University of Utah (Salt Lake City, UT) and Baylor College of Medicine (Houston, TX) and were in accordance with the Statement of the Association of Research for Vision and Ophthalmology for the Use of Animals in Ophthalmic and Vision Research.

\section{Immunohistochemistry}

The immunolabeling experiments were performed as previously described. ${ }^{8,19}$ Briefly, age-matched mouse eyes were immersion fixed for 2 hours using freshly prepared $4 \%$ para-

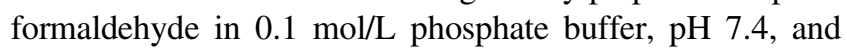
cryoprotected. Cryosections were cut sagittally (dorsal to ventral) through the optic nerve head and stained for different antibodies. Primary antibodies were applied to each group of two to four sections in a humidified chamber overnight at $4^{\circ} \mathrm{C}$, and were visualized with Alexa 488-, Alexa 647-, or
Cy3-conjugated secondary antibodies. The sections were viewed using a Zeiss LSM 510 or LSM 800 confocal microscope (Carl Zeiss Microscopy, Thornwood, NY). Primary antibodies against S-opsin, M-opsin, cone arrestin, G-protein-coupled receptor kinase 1 (GRK1), and cone transducin $\alpha$ subunit $\left(\mathrm{G}_{\alpha} \mathrm{t} 2\right)$ were described previously. ${ }^{8,19}$ Cone numbers from dorsal, central, and ventral retina were counted from four sections on the basis of arrestin staining. Data were expressed as the mean number of cones/field of view \pm SEM.

\section{Western Blot Analysis}

Retinal lysates from 1-month-old wild-type (WT) and mutant mice were used for Western blot analysis. Protein concentrations were measured by bicinchoninic acid assay. A total of $5 \mu \mathrm{g}$ retinal lysates was separated onto $10 \%$ SDSPAGE, transferred to a polyvinylidene difluoride membrane, and probed with primary antibodies against M-opsin, S-opsin, and glyceraldehyde-3-phosphate dehydrogenase, as described previously. ${ }^{8,19}$ The signals were visualized by the ChemiDoc imaging system (Bio-Rad, Hercules, CA).

\section{ERG Data}

Mice were dark adapted overnight before being anesthetized by i.p. injection of a combination of ketamine (65 to 100 $\mathrm{mg} / \mathrm{kg}$ ) and xylazine (10 to $20 \mathrm{mg} / \mathrm{kg}$ ). Pupils were dilated with $1 \%$ tropicamide. A corneal contact electrode was placed on the objective lens. Ground and reference electrodes were placed in the tail and head, respectively. Electroretinograms (ERGs) were recorded with the Phoenix
A
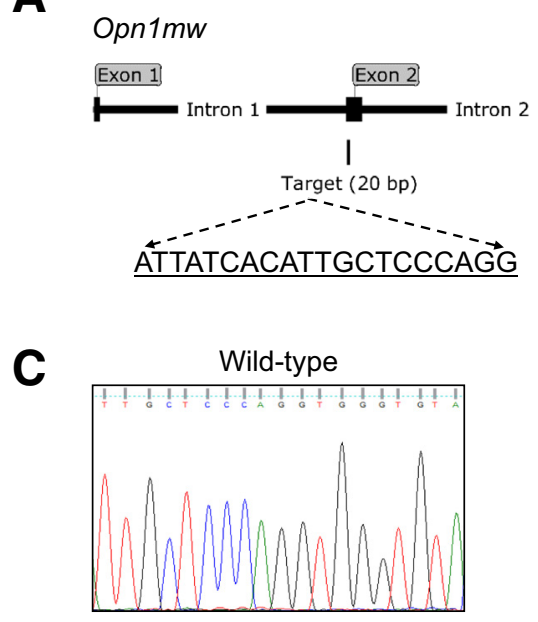

Opn1mw-

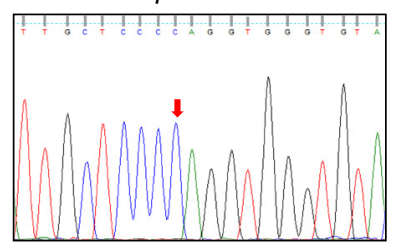

TTGCTCCCㄷAGGTGGGTGTA
B

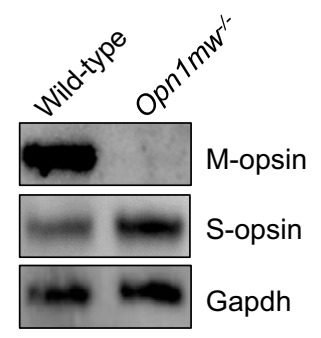

D

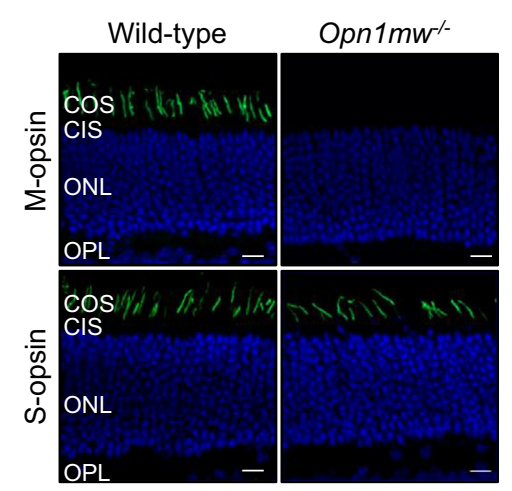

Figure 2 Generation and characterization of M-opsin gene deficient $\left(0 p n 1 \mathrm{mw}^{-/-}\right)$mice. A: A schematic illustration on the location of the single-guide RNA targeting sequence in the mouse Opn1mw gene. B: Western blot analysis of M-opsin and S-opsin from 1-month-old wildtype (WT) and Opn1mw- ${ }^{-1-}$ retinas. Glyceraldehyde-3phosphate dehydrogenase (Gapdh) was used as a loading control. C: DNA sequencing traces of WT and Opn1mw ${ }^{-1-}$ mice. There was a 1-bp insertion (arrow) at the targeting site, which caused a frameshift and truncation (Supplemental Figure S1). D: Immunolabeling of M-opsin and S-opsin in the retinal sections of 1-month-old WT and Opn $1 \mathrm{mw}^{-/-}$mice. M-opsin and S-opsin were imaged from the dorsal and ventral retina, respectively. Nuclei were stained with DAPI (blue). Scale bars $=10 \mu \mathrm{m}$. CIS, cone inner segment; $C O S$, cone outer segment; $0 \mathrm{NL}$, outer nuclear layer; $\mathrm{OPL}$, outer plexiform layer. 

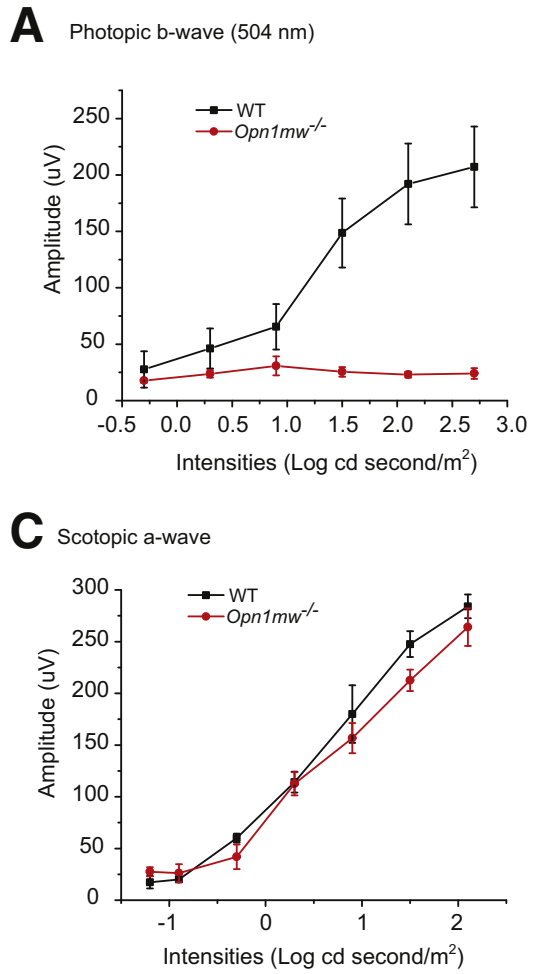

B Photopic b-wave $(360 \mathrm{~nm})$

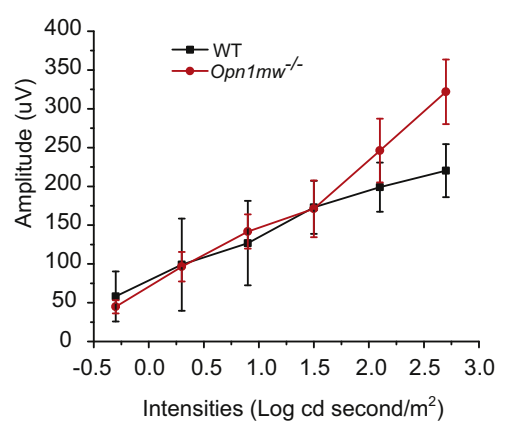

D Scotopic b-wave

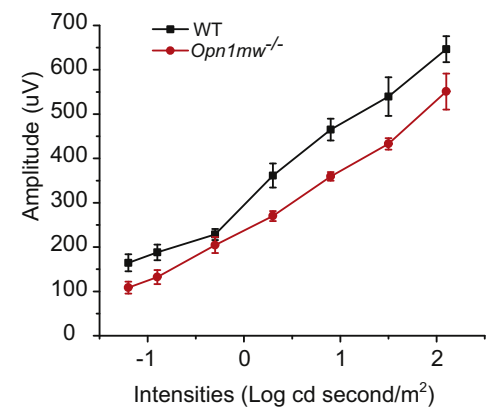

Figure 3 Photopic and scotopic electroretinography responses from 1-month-old M-opsin gene deficient $\left(0 p n 1 \mathrm{mw}^{-/-}\right)$and wild-type (WT) mice. The amplitudes of photopic b-wave responses to 504-nm (A) and 360-nm (B) stimuli at different light intensities $(-0.3,0.3,0.9$, 1.5, and $2.1 \log \mathrm{cd}$ second $\left./ \mathrm{m}^{2}\right)$. The scotopic a-wave amplitudes (C) and scotopic b-wave amplitudes (D) at different light intensities $(-1.2,-0.9,-0.3,0.3,0.9$, 1.5, and $2.1 \log \mathrm{cd}$ second $\left./ \mathrm{m}^{2}\right)$ to $504 \mathrm{~nm}$ light. Data are expressed as means \pm SEM. $n=4$ (A-D).
Ganzfeld ERG (Phoenix Research Labs, Pleasanton, CA). Flash stimuli intensities used were $-0.3,0.3,0.9,1.5$, and $2.1 \mathrm{log} \mathrm{cd}$ second $/ \mathrm{m}^{2}$ for photopic ERG, and $-1.2,-0.9$, $-0.3,0.3,0.9,1.5$, and $2.1 \log \mathrm{cd}$ second $/ \mathrm{m}^{2}$ for scotopic ERG. Five recordings were made with each intensity. Lightadapted responses were examined after bleaching at $2.1 \mathrm{log}$ cd second $/ \mathrm{m}^{2}$ with $504 \mathrm{~nm}$ light for 10 to 15 minutes. Four mice were used for recording in all conditions.

\section{Statistical Analysis}

All group results are expressed as means \pm SEM. Each experiment was performed at least three times for reproducibility. Comparisons between groups were made using the two-tailed $t$-test or one-way analysis of variance and Tukey post hoc tests for multiple groups. The Levene test was used to access homogeneity of variance. Values of $N$ were described in figure legends. Statistical analysis was performed with OriginPro 2016 version b9.3.2.303 (OriginLab Corporation, Northampton, MA). No statistical methods were used to predetermine the sample size, but our sample sizes are consistent with those generally used within the field. The mice were not randomized. $P<0.05$ was considered significant.

\section{Results}

Dorsal Cones in $\mathrm{Lrat}^{-/-}$Mice Exhibit Proteasome Stress

Previous studies have shown that M-opsin was degraded in Lrat $^{-1-}$ mice $^{8,19}$ and that M-opsin degradation was mediated by the proteasome but not lysosome pathway. ${ }^{21}$ This study hypothesized that the degradation of large amounts of mislocalized M-opsin can overload the ubiquitin-proteasome system and cause proteasome stress. To examine proteasomal insufficiency in $\mathrm{Lrat}^{-1-}$ cones, the $\mathrm{Lrat}^{-1-}$ mouse strain was crossed with a proteasome reporter mouse strain $U b^{G 76 V}$ GFP to generate $\mathrm{Lrat}^{-1-} U b^{G 76 V}-G F P$ mice. Accumulation of $\mathrm{Ub}^{\mathrm{G} 76 \mathrm{~V}}$ - green fluorescent protein (GFP), a cytoplasmic substrate carrying a degradation signal, indicates proteasomal insufficiency. ${ }^{29}$ The accumulation of $\mathrm{Ub}^{\mathrm{G} 76 \mathrm{~V}}-\mathrm{GFP}$ was detected by immunohistochemistry with an anti-GFP antibody. ${ }^{30}$ Cones from all regions of P18 $\mathrm{Lrat}^{-1-} \mathrm{Ub}^{G 76 V}-\mathrm{GFP}$ displayed robust reporter signal throughout cones [ie, from outer segment to synaptic terminal in green (cones were labeled with rhodamine-peanut agglutinin in red)] (Figure 1). No cone-specific GFP signal was detected in control $U b^{G 76 V}$. GFP. Proteasomal insufficiency in central/ventral cones was likely caused by aggregated $\mathrm{S}$-opsin-mediated proteasome inhibition. ${ }^{31-34}$ Proteasome insufficiency in dorsal cones was likely caused by M-opsin degradation-induced proteasome overload.

\section{Genetic Deletion of M-Opsin from $\mathrm{Lrat}^{-/-} \mathrm{Opn1sw} \mathrm{s}^{-/-}$ Mice Prevents M Cone Degeneration}

To overcome the difficulties associated with the mixed expression of $\mathrm{M}$-opsin and $\mathrm{S}$-opsin in mouse cones and the rapid degeneration caused by S-opsin in $\mathrm{Lrat}^{-1-}$ mice, the $\mathrm{Lrat}^{-1-} \mathrm{Opn} 1 \mathrm{sw}^{-1-}$ mice were generated as a pure $\mathrm{M}$ cone LCA model to study the role of M-opsin in cone 
A
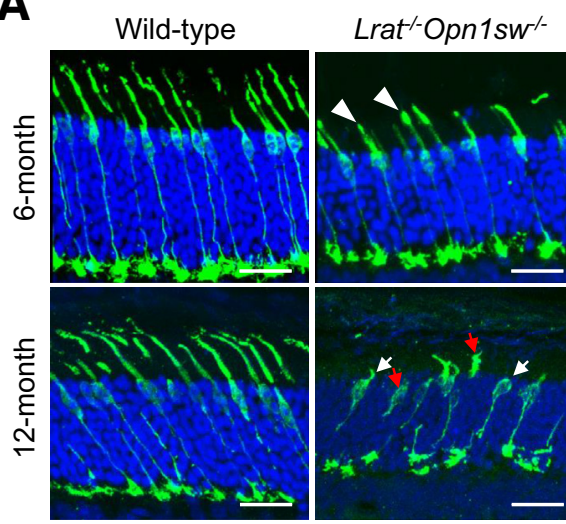

B

6-month

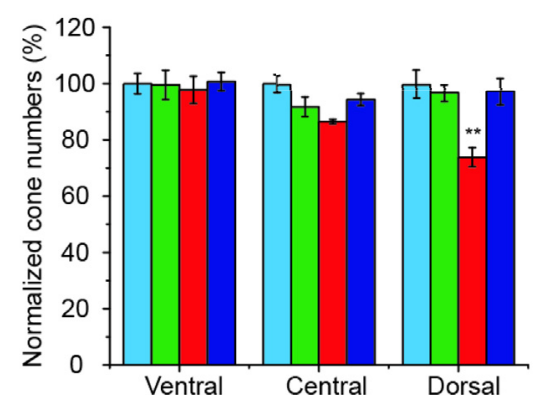

C

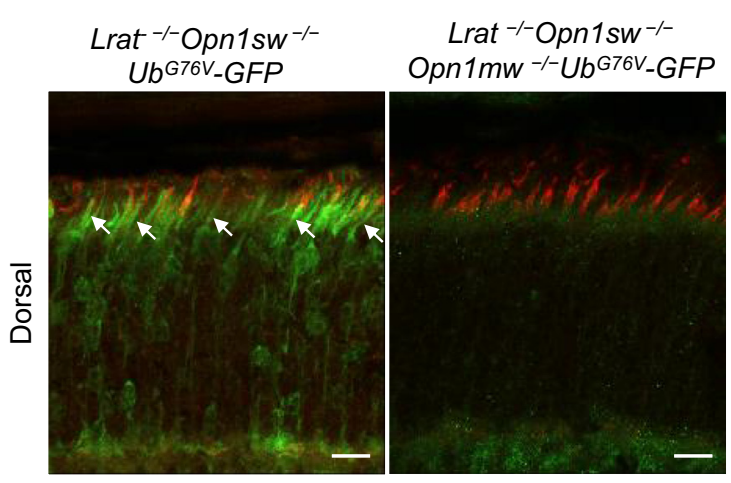

12-month

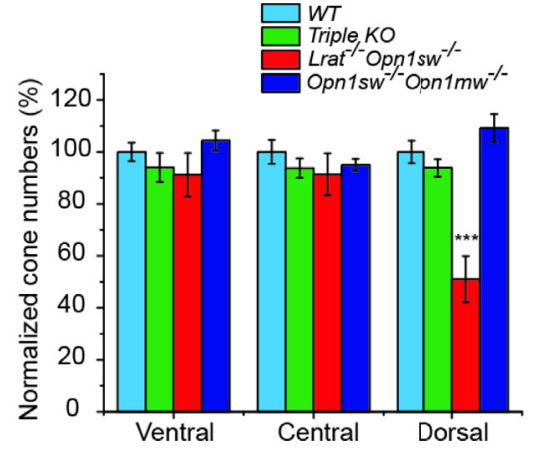

Triple KO

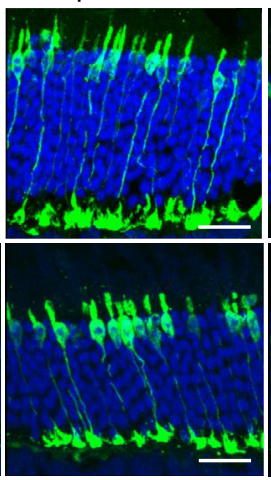
Opn1sw/-Opn1mw
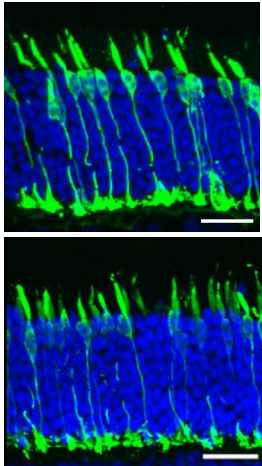

Central

$U b^{G 76 V-G F P}$

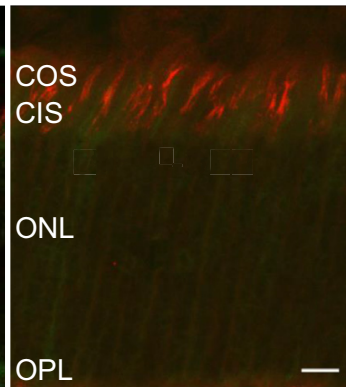

Figure 4 M-opsin deletion prevents dorsal $M$ cone degeneration in $\mathrm{Lrat}^{-/-} \mathrm{Opn} 1 \mathrm{sw}^{-/-} \mathrm{Opn} 1 \mathrm{mw}^{-/-}$ mice. A: Retinal sections from 6-month-old and 12-month-old wild-type (WT), $\mathrm{Lrat}^{-/} \mathrm{Opn1sw}^{-/}$, Lrat $^{-/-}$Opn1sw ${ }^{-/-}$Opn1mw $w^{-/-}$[triple knockout (KO)], and Opn1sw ${ }^{-1-} O p n 1 \mathrm{mw}^{-/-}$mice were labeled with anti-mouse cone arrestin antibody (in green). White arrowheads indicate unhealthy dorsal cones from 6-month-old $\mathrm{Lrat}^{-/-} \mathrm{Opn} 1 \mathrm{sw}^{-/-}$mice with swollen outer and inner segments; white arrows, cones from 12-month-old $\mathrm{Lrat}^{-/-} \mathrm{Opnn}_{\mathrm{sw}}{ }^{-/-}$mice that had lost outer segments; red arrows, cones from 12-month-old $\mathrm{Lrat}^{-/-} \mathrm{Opn1Sw}^{-1-}$ mice with broken/fragmented structure. Representative retinal images from the dorsal regions of the indicated genotype are shown. Nuclei were stained with DAPI. B: Quantitative results of relative cone numbers at the ventral, central, and dorsal retina from the indicated genotype. Data were normalized to WT dorsal region. C: $M$-opsin deletion reduces proteasomal stress in dorsal $\mathrm{M}$ cones in $\mathrm{Lrat}^{-/-} \mathrm{Opn1sw}{ }^{-/-}$mice. The reporter $\mathrm{Ub}^{\mathrm{G} 76 \mathrm{~V}}$-green fluorescent protein (GFP) signal from the dorsal retinas of $\mathrm{P} 18 \mathrm{Lrat}^{-/-}$ Opn1sw ${ }^{-/-} U b^{G 76 V}-G F P$ mice, Lrat $^{-/-} \mathrm{Opn}_{\mathrm{S}} \mathrm{sw}^{-/-}$ Opn1mw $w^{-1-} U b^{G 76 V}-G F P$, and $U b^{G 76 V}-G F P$ control mice were labeled with an anti-GFP antibody (in green). Cones were labeled with rhodamine-PNA (in red). Arrows indicate dorsal cones from $\mathrm{Lrat}^{-/-}$ Opn1sw ${ }^{-/} U b^{G 76 V}-$ GFP mice that displayed GFP signal. Data are expressed as means \pm SEM (B). $n=4$ to 7 for 6 -month samples (B); $n=4$ to 6 for each genotype for 12 -month samples (B). ${ }^{* *} P<0.01$, $* * * P<0.001$ versus all other three genotypes at both 6 and 12 months (one-way analysis of variance with Tukey post hoc analysis). Scale bars: $20 \mu \mathrm{m}$ (A); $10 \mu \mathrm{m}$ (C). CIS, cone inner segment; COS, cone outer segment; $\mathrm{ONL}$, outer nuclear layer; $\mathrm{OPL}$, outer plexiform layer. degeneration. First, Opn $1 m w^{-1-}$ mice were generated by CRISPR-Cas9. Opn1mw was disrupted by one nucleotide insertion in the second exon, which causes a frameshift and truncation at the second exon (Figure 2, A and C, and Supplemental Figure S1). Since the gene OpnImw is on the $\mathrm{X}$ chromosome, both male Opnlmw $w^{Y /-}$ and female OpnImw $w^{-1-}$ mice are referred to as Opn $1 m w^{-1-}$ mice for convenience hereafter. Both male and female Opn $1 \mathrm{mw}^{-1-}$ mice have normal body size and fertility. Immunohistochemistry and Western blot analysis confirmed the absence of M-opsin in the retina of Opn1mw ${ }^{-1-}$ mice (Figure 2, B and D). The expression level of S-opsin was increased in Opnlmw $w^{-1-}$ mice (Figure 2B), which mirrors the result of M-opsin up-regulation in $S$-opsin ${ }^{-1-}$ mice due to transcriptional compensation. $^{28}$ In photopic ERG recordings, Opn $1 \mathrm{mw}^{-l-}$ mice lost light sensitivity at $504 \mathrm{~nm}$ light and only responded to $360 \mathrm{~nm}$ light, which provides functional validation on M-opsin deletion (Figure 3, A and B). The enhanced photopic responses at $360 \mathrm{~nm}$ at high intensities compared with WT is consistent with the increased S-opsin expression (Figure 3B). Scotopic ERG reflects rod input at low intensities $(<-0.85 \log$ cd second/ $\left.\mathrm{m}^{2}\right)^{35}$ and mixed rod and cone input at high intensities. Compared with WT mice, Opn1mw ${ }^{-1-}$ mice showed decreased scotopic a- and b-wave responses at high light intensities to $504 \mathrm{~nm}$ light because of the absence of M-opsin-mediated cone responses (Figure 3, C and D). The OpnImw ${ }^{-1-}$ mice were bred with $\mathrm{Lrat}^{-1-} \mathrm{OpnIsw}^{-1-}$ mice 


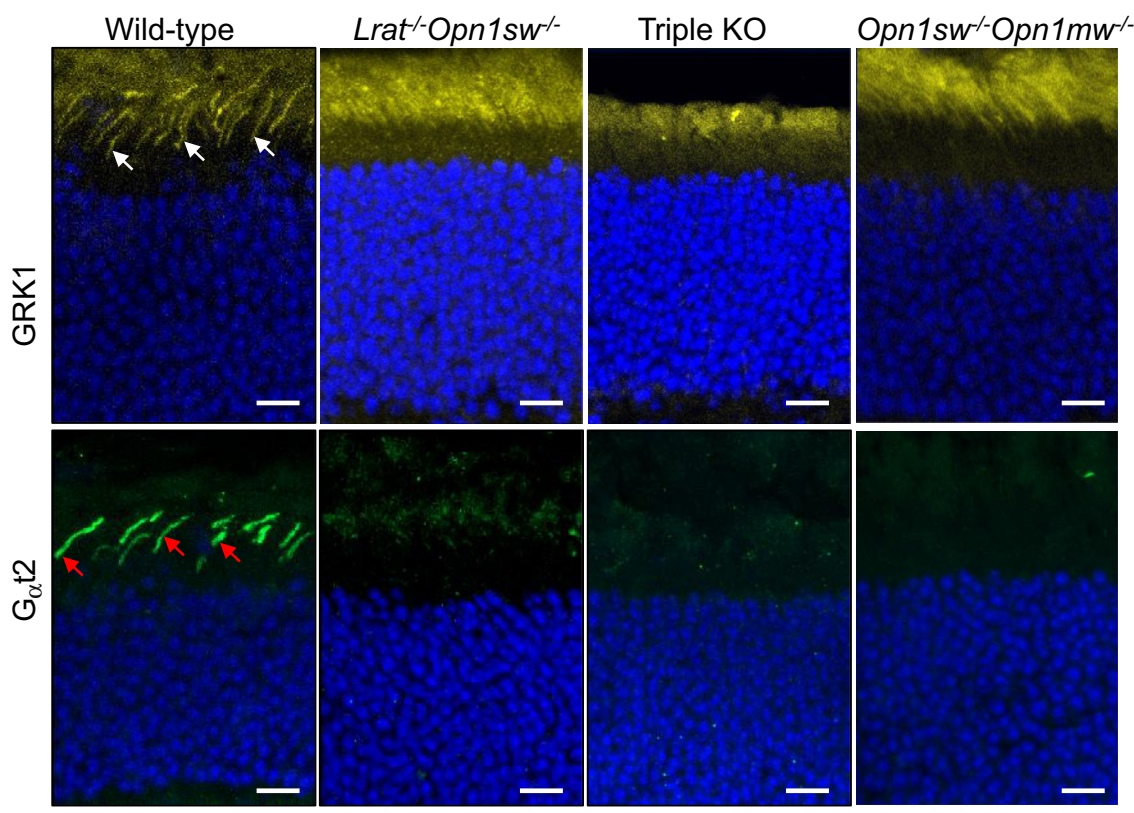

Figure 5 Immunolocalization of cone membrane-associated proteins G-protein-coupled receptor kinase 1 (GRK1) and cone transducin $\alpha$ subunit (G, $\mathrm{t} 2)$ in wild-type (WT), $\mathrm{Lrat}^{-/-}$ Opn1sw ${ }^{-/-}, \mathrm{Lrat}^{-/-} \mathrm{Opn} 1 \mathrm{sw} \mathrm{w}^{-/-} \mathrm{Opn1mw^{-/- }}$ [triple knockout (KO)], and Opn1sw ${ }^{-/}$Opn1 $1 \mathrm{mw}^{-/-}$mice. One-month-old mouse retinal sections were stained with antibodies against GRK1 (in yellow) and $\mathrm{G}_{\alpha} \mathrm{t} 2$ (green). White arrows GRK1 signal in WT cones; red arrows, $\mathrm{G}_{\alpha} \mathrm{t} 2$ signal in WT cones. Nuclei were stained with DAPI (blue). Scale bars $=10 \mu \mathrm{m}$.

to generate $\mathrm{Lrat}^{-/-} \mathrm{OpnI} \mathrm{sw}^{-1-} \mathrm{OpnImw}^{-/-}$mice. Dorsal cones of $\mathrm{Lrat}^{-1-} \mathrm{Opnlsw}^{-1-}$ mice are morphologically unhealthy at 6 months, with swollen outer and inner segments (Figure 4A). This became much worse at 12 months. Most remaining cones have lost their outer segments (Figure 4A) with broken or fragmented structure (Figure 4A). Compared with WT, $\mathrm{Lrat}^{-1-} \mathrm{OpnI} \mathrm{sw}^{-1-}$ mice have lost 26\% $(P<0.01)$ and $49 \%(P<0.001)$ cones at 6 and 12 months, respectively (Figure 4B). In contrast, 97\% and 94\% dorsal cones survived in $\mathrm{Lrat}^{-1-} \mathrm{Opnlsw}^{-1-} \mathrm{OpnImw}^{-1-}$ mice at 6 and 12 months, respectively, which is not significantly different from control WT and Opn1sw ${ }^{-1-}$ Opn1mw $w^{-1-}$ mice, suggesting that $\mathrm{M}$-opsin plays a major role in dorsal cone degeneration in $\mathrm{Lrat}^{-1-}$ mice. Both $\mathrm{Lrat}^{-1-} \mathrm{Opnl} \mathrm{sw}^{-1-}$ OpnImw ${ }^{-1-}$ and Opn1sw ${ }^{-1-}$ OpnImw ${ }^{-1-}$ cones are shorter than WT cones because of the lack of normal outer segments resulting from cone opsin deletions. In fact, only cone inner segments can be clearly detected by double labeling of cone arrestin (in green) and peanut agglutinin (in red) (Supplemental Figure S2). This is in contrast to the presence of both outer segments (Supplemental Figure S2, indicating strong colocalization signal between cone arrestin and peanut agglutinin, note peanut agglutinin labels cone matrix sheath) and inner segments (Supplemental Figure S2) in WT cones. Previous work has shown that cones can survive with little cone opsins (ie, ventral cones in Opnl sw ${ }^{-1-}$ and $\mathrm{Lrat}^{-1-} \mathrm{Opnl} \mathrm{sw}^{-1-}$ mice $)^{19,28,36,37}$; this study provides conclusive evidence that cones can survive with no cone opsins (ie, 6- and 12-month Opn1sw ${ }^{-1-}$ Opn1mw ${ }^{-1-}$ cones), which is different from rods that degenerate rapidly without rhodopsin. ${ }^{38}$ It was shown previously that deletion of S-opsin prevents ventral and central cone degeneration in $\mathrm{Lrat}^{-/-} \mathrm{Opnl} \mathrm{sw}^{-/-}$mice up to 12 months. ${ }^{19}$ This result is confirmed in Figure 4B.
Genetic Deletion of M-Opsin from $\mathrm{Lrat}^{-/-} \mathrm{Opn1sw} \mathrm{s}^{-/-}$ Mice Reduces Proteasome Stress in M Cones

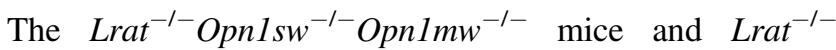
Opn1sw ${ }^{-1-}$ mice were bred with $U b^{G 76 V}$-GFP to generate $\mathrm{Lrat}^{-1-} \mathrm{Opn}_{\mathrm{sw}}{ }^{-1-} \mathrm{Opn}_{\mathrm{Imw}}{ }^{-1-} \mathrm{Ub} \mathrm{b}^{G 76 V}-\mathrm{GFP}$ mice and $\mathrm{Lrat}^{-1-}$ Opn1sw ${ }^{-1-} U b^{G 76 V}$-GFP mice to assess the role of proteasome stress in M-cone degeneration. In P18 $\mathrm{Lrat}^{-1-}$ Opn1sw ${ }^{-1-} U b^{G 76 V}-$ GFP mice, dorsal cones displayed strong GFP signal, indicating proteasome inefficiency (Figure 4C). In contrast, dorsal cones from P18 Lrat $^{-1-}$ Opn1sw ${ }^{-1-}$ Opn1mw ${ }^{-1-} U b^{G 76 V}$-GFP mice exhibited markedly decreased GFP signal, suggesting M-opsin degradation plays a major role for proteasome stress in $\mathrm{M}$ cones. Interestingly, GFP signal in the ventral and central cones in $\mathrm{Lrat}^{-1-}{ }^{-} \mathrm{Opnl} 1 \mathrm{sw}^{-1-} \mathrm{Opn} 1 \mathrm{mw} \mathrm{w}^{-1-} \mathrm{Ub}^{G 76 V}{ }_{-G F P}$ mice was also decreased compared with those of $\mathrm{Lrat}^{-1-} \mathrm{Opnl} \mathrm{sw}^{-1-} \mathrm{Ub}^{G 76 \mathrm{~V}}$. GFP mice (Supplemental Figure S3). One explanation is that in the absence of S-opsin, M-opsin is increased because of transcriptional compensation and causes proteasome stress in central and ventral cones. ${ }^{28}$ The residual GFP signal in $\mathrm{Lrat}^{-1-} \mathrm{Opnlsw}^{-1-} \mathrm{Opn} 1 \mathrm{mw} \mathrm{w}^{-1-} \mathrm{Ub}^{\mathrm{G} 76 V}{ }_{-} \mathrm{GFP}$ mice compared with WT mice is likely caused by proteasome stress associated with the degradation of other cone phototransduction proteins (see below).

\section{Mistargeting of Membrane-Associated Proteins in Lrat $^{-/-}$Opn1sw ${ }^{-/-}$Opn1mw ${ }^{-/-}$Cones}

Several membrane-associated proteins (eg, M/S opsins, GRK1, and $\mathrm{G}_{\alpha} \mathrm{t} 2$ ) involved in cone phototransduction fail to traffic to the outer segment of $\mathrm{Lrat}^{-1-}$ or Rpe $65^{-/-}$cones properly. ${ }^{7}$ The mistrafficked proteins are degraded through 


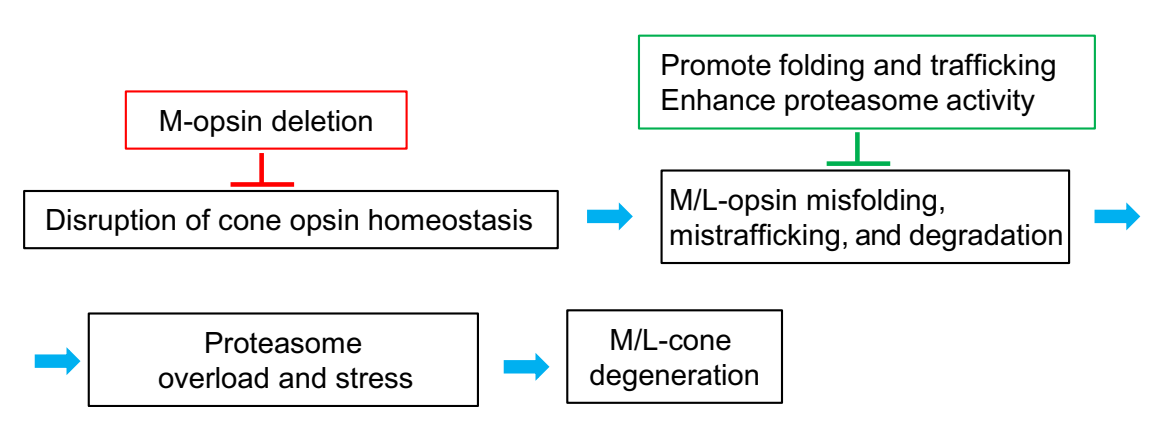

Figure 6 A schematic on the role of M-opsin in multiple forms of cone degeneration. Mislocalized M/L-opsins are degraded, which causes proteasome stress and dorsal $M$ cone degeneration. Deletion of $M$-opsin prevents dorsal $M$ cone degeneration. One strategy to protect $M / L$ cones from degeneration is to promote the folding and targeting of $\mathrm{M} / \mathrm{L}-\mathrm{opsins}$ and to enhance proteasome activity. a post-translational mechanism, ${ }^{7}$ except that S-opsin aggregates and resists proteasome degradation. ${ }^{8}$ The subcellular localization of GRK1 and $\mathrm{G}_{\alpha} \mathrm{t} 2$ in the retinas of

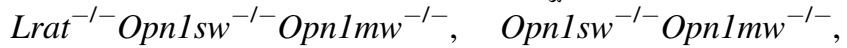
$\mathrm{Lrat}^{-1-} \mathrm{OpnI}_{\mathrm{sw}}{ }^{-1-}$, and WT mice was examined. GRK1 was expressed in both rods and cones (Figure 5) in WT. In sharp contrast, no GRK1 signal was detected in cones in $\mathrm{Lrat}^{-1-}{ }^{-} \mathrm{Opn1} \mathrm{sw}^{-1-} \mathrm{OpnImw}^{-1-}$, Opn1sw ${ }^{-1-} \mathrm{OpnImw}^{-1-}$, and $\mathrm{Lrat}^{-1-} \mathrm{Opnl}_{\mathrm{sw}}{ }^{-1-}$ mice (Figure 5). $\mathrm{G}_{\alpha} \mathrm{t} 2$ was markedly reduced in $\mathrm{Lrat}^{-1-} \mathrm{Opnlsw}^{-1-}$ mice (Figure 5), as shown previously, ${ }^{8}$ and was undetectable in Lrat $^{-1-}$ OpnIsw $^{-1-}$ OpnImw $^{-1-}$ and Opn1sw ${ }^{-1}$ OpnImw $^{-1-}$ mice (Figure 5) compared with the robust signal in WT cones (Figure 5). The prevention of dorsal $\mathrm{M}$ cone degeneration in $\mathrm{Lrat}^{-/}{ }^{-} \mathrm{Opnl} \mathrm{sw}^{-1-} \mathrm{OpnImw}^{-/-}$mice and the continued degradation of many cone outer segment proteins in this mouse strain suggest that the degradation of many cone membrane-associated proteins other than $\mathrm{M}$ opsin does not play a significant role in cone viability, likely because of their substantially smaller quantities than M-opsin (see more in Discussion).

\section{Discussion}

The major finding of this study is that deletion of M-opsin relieved proteasome stress and prevented the dorsal $\mathrm{M}$ cone degeneration in $\mathrm{Lrat}^{-1-} \mathrm{Opnl} \mathrm{sw}^{-1-}$ mice for at least 1 year. In the absence of 11-cis-retinal, daily degradation of large amounts of M-opsin could overload the proteasome and cause chronic proteasomal insufficiency, which may lead to the accumulation of misfolded proteins in the ER by inhibiting ER-associated degradation and triggering chronic ER stress in M cones. On the other hand, chronic proteasomal insufficiency may also affect normal cellular function. ${ }^{39,40}$ Indeed, proteasome overload or impairment has been implicated in several types of rod degeneration associated with protein misfolding or mistargeting. ${ }^{23,41-43}$ To the knowledge of the authors, this is the first study to examine the role of proteasome stress in cone degeneration.
Because several mouse models of cone dystrophy with cone opsin mislocalization exhibited M-opsin degradation and slower dorsal cone degeneration [eg, cone cyclic nucleotidegated channel A subunit (Cnga3) knockout, ${ }^{44}$ guanylate cyclase 1 (Gucy2e) knockout (LCA1), ${ }^{45,46}$ rd3 (LCA12), ${ }^{47}$ Aipll knockout $(L C A 4),{ }^{48}$ and kinesin $3 \mathrm{~A}$ (Kif3a) knockout in cones], ${ }^{49} \mathrm{M}$-opsin degradation associated proteasome stress may represent a general mechanism for dorsal cone degeneration in these models (Figure 6).

In addition to M-opsin, outer segment proteins, such as $\mathrm{G}_{\alpha} \mathrm{t} 2$ and GRK1, are also degraded in $\mathrm{Lrat}^{-1-} \mathrm{Opn} 1 \mathrm{sw}^{-1-}$ cones. Previous studies have shown that guanylate cyclase 1 and cone phosphodiesterase $6 \alpha^{\prime}$ were degraded in $\mathrm{Lrat}^{-1-}$ cones. $^{7}$ The degradation of these proteins should also cause proteasome stress. Why did deletion of $\mathrm{M}$-opsin alone from Lrat $^{-1-} \mathrm{Opnlsw}^{-1-}$ mice substantially reduce proteasome stress (Figure 4C) and completely prevent dorsal $\mathrm{M}$ cone degeneration? The reason is likely because of the huge difference in protein quantity between cone opsins and other outer segment proteins. For example, the molecular ratio between rhodopsin/G-protein is 10:1, rhodopsin/phosphodiesterase is 50:1, and rhodopsin/GRK1 is 500:1 in mammalian rods. ${ }^{50}$ A similar ratio can be expected between cone opsins and various outer segment proteins. The dominance of cone opsins over other proteins explains why it plays a determined role in proteasome stress and cone degeneration in LCA.

The most promising treatment for LRAT or RPE65 LCA is to use gene augmentation therapy with a normal copy of LRAT (or RPE65) to restore the visual cycle. In 2017, voretigene neparvovec (Luxturna) was approved by the Food and Drug Administration for the treatment of RPE65LCA (LCA2), making it the first directly administered gene therapy approved in the United States that targets a genetic disease caused by mutations in a specific gene. However, there were reports that photoreceptors continued to degenerate and vision benefit faded within 3 years despite initial vision gain after successful adeno-associated virus (AAV)RPE65 therapy. ${ }^{11,15,16}$ In addition, there was no improvement in foveal cone function despite AAV vectors having 
been delivered to the fovea in most participants. ${ }^{16,51}$ These studies highlight the importance of understanding the mechanism of foveal cone degeneration and designing adjunct therapy to slow photoreceptor degeneration in LCA patients in future clinical gene therapy trials. The prominent role of cone opsins in cone degeneration suggests that, in addition to restoring the 11-cis-retinal supply, research needs to focus on the folding, trafficking, and degradation properties of cone opsins to design an effective prevention and treatment strategy. This work demonstrated the importance of M-opsin degradation associated proteasome stress in $\mathrm{L} / \mathrm{M}$ cone (the dominant cone type in the fovea). This study proposes to design drugs to help the folding and targeting of M/L-opsins and to enhance proteasome activity to slow down cone degeneration as an adjunct treatment for gene therapy to achieve the best therapeutic efficacy for LCA (Figure 6).

\section{Acknowledgments}

We thank Drs. Wolfgang Baehr, Edward N. Pugh Jr, and Jeannie Chen for providing the $\mathrm{Lrat}^{-1-}$ mice and the cone arrestin antibody, the Opn1sw ${ }^{-1-}$ mice, and the S-opsin antibody, respectively.

\section{Author Contributions}

H.X. performed most experiments, analyzed the data, and wrote the initial draft; N.E. collected samples; X.Z. maintained and genotyped mice; O.Z. performed genotyping; Y.F. designed the study, analyzed the data, and wrote the manuscript.

\section{Supplemental Data}

Supplemental material for this article can be found at http://doi.org/10.1016/j.ajpath.2020.01.005.

\section{References}

1. Jacobson SG, Aleman TS, Cideciyan AV, Heon E, Golczak M, Beltran WA, Sumaroka A, Schwartz SB, Roman AJ, Windsor EA, Wilson JM, Aguirre GD, Stone EM, Palczewski K: Human cone photoreceptor dependence on RPE65 isomerase. Proc Natl Acad Sci U S A 2007, 104:15123-15128

2. Maeda T, Cideciyan AV, Maeda A, Golczak M, Aleman TS, Jacobson SG, Palczewski K: Loss of cone photoreceptors caused by chromophore depletion is partially prevented by the artificial chromophore pro-drug, 9-cis-retinyl acetate. Hum Mol Genet 2009, 18: $2277-2287$

3. Redmond TM, Yu S, Lee E, Bok D, Hamasaki D, Chen N, Goletz P, Ma JX, Crouch RK, Pfeifer K: Rpe65 is necessary for production of 11-cis-vitamin A in the retinal visual cycle. Nat Genet 1998, 20: 344-351

4. Batten ML, Imanishi Y, Maeda T, Tu DC, Moise AR, Bronson D, Possin D, Van Gelder RN, Baehr W, Palczewski K: Lecithin-retinol acyltransferase is essential for accumulation of all-trans-retinyl esters in the eye and in the liver. J Biol Chem 2004, 279:10422-10432
5. Fan J, Rohrer B, Frederick JM, Baehr W, Crouch RK: Rpe65-/- and Lrat-/- mice: comparable models of leber congenital amaurosis. Invest Ophthalmol Vis Sci 2008, 49:2384-2389

6. Rohrer B, Lohr HR, Humphries P, Redmond TM, Seeliger MW, Crouch RK: Cone opsin mislocalization in Rpe65-/- mice: a defect that can be corrected by 11-cis retinal. Invest Ophthalmol Vis Sci 2005, 46:3876-3882

7. Zhang H, Fan J, Li S, Karan S, Rohrer B, Palczewski K, Frederick JM, Crouch RK, Baehr W: Trafficking of membraneassociated proteins to cone photoreceptor outer segments requires the chromophore 11-cis-retinal. J Neurosci 2008, 28:4008-4014

8. Zhang T, Zhang N, Baehr W, Fu Y: Cone opsin determines the time course of cone photoreceptor degeneration in Leber congenital amaurosis. Proc Natl Acad Sci U S A 2011, 108:8879-8884

9. Pang J, Boye SE, Lei B, Boye SL, Everhart D, Ryals R, Umino Y, Rohrer B, Alexander J, Li J, Dai X, Li Q, Chang B, Barlow R, Hauswirth WW: Self-complementary AAV-mediated gene therapy restores cone function and prevents cone degeneration in two models of Rpe65 deficiency. Gene Ther 2010, 17:815-826

10. Chen Y, Moiseyev G, Takahashi Y, Ma JX: RPE65 gene delivery restores isomerohydrolase activity and prevents early cone loss in Rpe65-/- mice. Invest Ophthalmol Vis Sci 2006, 47:1177-1184

11. Cideciyan AV, Jacobson SG, Beltran WA, Sumaroka A, Swider M, Iwabe S, Roman AJ, Olivares MB, Schwartz SB, Komaromy AM, Hauswirth WW, Aguirre GD: Human retinal gene therapy for Leber congenital amaurosis shows advancing retinal degeneration despite enduring visual improvement. Proc Natl Acad Sci U S A 2013, 110: E517-E525

12. Bainbridge JW, Smith AJ, Barker SS, Robbie S, Henderson R, Balaggan K, Viswanathan A, Holder GE, Stockman A, Tyler N, Petersen-Jones S, Bhattacharya SS, Thrasher AJ, Fitzke FW, Carter BJ, Rubin GS, Moore AT, Ali RR: Effect of gene therapy on visual function in Leber's congenital amaurosis. N Engl J Med 2008, 358:2231-2239

13. Maguire AM, Simonelli F, Pierce EA, Pugh EN Jr, Mingozzi F, Bennicelli J, Banfi S, Marshall KA, Testa F, Surace EM, Rossi S, Lyubarsky A, Arruda VR, Konkle B, Stone E, Sun J, Jacobs J, Dell'Osso L, Hertle R, Ma JX, Redmond TM, Zhu X, Hauck B, Zelenaia O, Shindler KS, Maguire MG, Wright JF, Volpe NJ, McDonnell JW, Auricchio A, High KA, Bennett J: Safety and efficacy of gene transfer for Leber's congenital amaurosis. N Engl J Med 2008, 358:2240-2248

14. Cideciyan AV, Aleman TS, Boye SL, Schwartz SB, Kaushal S, Roman AJ, Pang JJ, Sumaroka A, Windsor EA, Wilson JM, Flotte TR, Fishman GA, Heon E, Stone EM, Byrne BJ, Jacobson SG, Hauswirth WW: Human gene therapy for RPE65 isomerase deficiency activates the retinoid cycle of vision but with slow rod kinetics. Proc Natl Acad Sci U S A 2008, 105:15112-15117

15. Jacobson SG, Cideciyan AV, Roman AJ, Sumaroka A, Schwartz SB, Heon E, Hauswirth WW: Improvement and decline in vision with gene therapy in childhood blindness. N Engl J Med 2015, 372: 1920-1926

16. Bainbridge JWB, Mehat MS, Sundaram V, Robbie SJ, Barker SE, Ripamonti C, Georgiadis A, Mowat FM, Beattie SG, Gardner PJ, Feathers KL, Luong VA, Yzer S, Balaggan K, Viswanathan A, de Ravel TJL, Casteels I, Holder GE, Tyler N, Fitzke FW, Weleber RG, Nardini M, Moore AT, Thompson DA, Petersen-Jones SM, Michaelides M, van den Born LI, Stockman A, Smith AJ, Rubin G, Ali RR: Long-term effect of gene therapy on Leber's congenital amaurosis. N Engl J Med 2015, 372:1887-1897

17. Gardiner KL, Cideciyan AV, Swider M, Dufour VL, Sumaroka A, Komáromy AM, Hauswirth WW, Iwabe S, Jacobson SG, Beltran WA, Aguirre GD: Long-term structural outcomes of latestage RPE65 gene therapy. Mol Ther 2020, 28:266-278

18. Znoiko SL, Rohrer B, Lu K, Lohr HR, Crouch RK, Ma JX: Downregulation of cone-specific gene expression and degeneration of cone photoreceptors in the Rpe65-/- mouse at early ages. Invest Ophthalmol Vis Sci 2005, 46:1473-1479 
19. Zhang T, Enemchukwu NO, Jones A, Wang S, Dennis E, Watt CB, Pugh EN, Fu Y: Genetic deletion of S-opsin prevents rapid cone degeneration in a mouse model of Leber congenital amaurosis. Hum Mol Genet 2015, 24:1755-1763

20. Williamson SJ, Cummins HZ: Light and Color in Nature and Art. New York, NY, John Wiley and Sons, 1983

21. Sato K, Ozaki T, Ishiguro S, Nakazawa M: M-opsin protein degradation is inhibited by MG-132 in Rpe65 ${ }^{-1-}$ retinal explant culture. Mol Vis 2012, 18:1516-1525

22. Wang $\mathrm{T}$, Chen J: Induction of the unfolded protein response by constitutive G-protein signaling in rod photoreceptor cells. J Biol Chem 2014, 289:29310-29321

23. Lobanova ES, Finkelstein S, Skiba NP, Arshavsky VY: Proteasome overload is a common stress factor in multiple forms of inherited retinal degeneration. Proc Natl Acad Sci U S A 2013, 110: 9986-9991

24. Chiang W-C, Kroeger H, Sakami S, Messah C, Yasumura D, Matthes MT, Coppinger JA, Palczewski K, LaVail MM, Lin JH: Robust endoplasmic reticulum-associated degradation of rhodopsin precedes retinal degeneration. Mol Neurobiol 2015, 52:679-695

25. Ando R, Noda K, Tomaru U, Kamoshita M, Ozawa Y, Notomi S, Hisatomi T, Noda M, Kanda A, Ishibashi T, Kasahara M, Ishida S: Decreased proteasomal activity causes photoreceptor degeneration in mice. Invest Ophthalmol Vis Sci 2014, 55:4682-4690

26. Lobanova ES, Finkelstein S, Li J, Travis AM, Hao Y, Klingeborn M, Skiba NP, Deshaies RJ, Arshavsky VY: Increased proteasomal activity supports photoreceptor survival in inherited retinal degeneration. Nat Commun 2018, 9:1738

27. Ran FA, Hsu PD, Wright J, Agarwala V, Scott DA, Zhang F: Genome engineering using the CRISPR-Cas9 system. Nat Protoc 2013, 8:2281-2308

28. Daniele LL, Insinna C, Chance R, Wang J, Nikonov SS, Pugh EN: A mouse $\mathrm{M}$-opsin monochromat: retinal cone photoreceptors have increased M-opsin expression when S-opsin is knocked out. Vision Res 2011, 51:447-458

29. Lindsten K, Menendez-Benito V, Masucci MG, Dantuma NP: A transgenic mouse model of the ubiquitin/proteasome system. Nat Biotechnol 2003, 21:897-902

30. Cheroni C, Marino M, Tortarolo M, Veglianese P, De Biasi S, Fontana E, Zuccarello LV, Maynard CJ, Dantuma NP, Bendotti C: Functional alterations of the ubiquitin-proteasome system in motor neurons of a mouse model of familial amyotrophic lateral sclerosis. Hum Mol Genet 2009, 18:82-96

31. Thibaudeau TA, Anderson R, Smith DM: A common mechanism of proteasome impairment by neurodegenerative disease-associated oligomers. FASEB J 2017, 31:763.8

32. Kristiansen M, Deriziotis P, Dimcheff DE, Jackson GS, Ovaa H, Naumann H, Clarke AR, van Leeuwen FWB, Menéndez-Benito V, Dantuma NP, Portis JL, Collinge J, Tabrizi SJ: Disease-associated prion protein oligomers inhibit the 26S proteasome. Mol Cell 2007, 26:175-188

33. Snyder H, Mensah K, Theisler C, Lee J, Matouschek A, Wolozin B: Aggregated and monomeric alpha-synuclein bind to the S6' proteasomal protein and inhibit proteasomal function. J Biol Chem 2003, 278:11753-11759

34. Chen L, Thiruchelvam MJ, Madura K, Richfield EK: Proteasome dysfunction in aged human alpha-synuclein transgenic mice. Neurobiol Dis 2006, 23:120-126

35. Lei B, Yao G, Zhang K, Hofeldt KJ, Chang B: Study of rod- and cone-driven oscillatory potentials in mice. Invest Ophthalmol Vis Sci 2006, 47:2732-2738

36. Deng W-T, Li J, Zhu P, Chiodo VA, Smith WC, Freedman B, Baehr W, Pang J, Hauswirth WW: Human L- and M-opsins restore
M-cone function in a mouse model for human blue cone monochromacy. Mol Vis 2018, 24:17-28

37. Deng W-T, Li J, Zhu P, Freedman B, Smith WC, Baehr W, Hauswirth WW: Rescue of M-cone function in aged Opn1mw-/mice, a model for late-stage blue cone monochromacy. Invest Ophthalmol Vis Sci 2019, 60:3644-3651

38. Lem J, Krasnoperova NV, Calvert PD, Kosaras B, Cameron DA, Nicolo M, Makino CL, Sidman RL: Morphological, physiological, and biochemical changes in rhodopsin knockout mice. Proc Natl Acad Sci U S A 1999, 96:736-741

39. Dantuma NP, Bott LC: The ubiquitin-proteasome system in neurodegenerative diseases: precipitating factor, yet part of the solution. Front Mol Neurosci 2014, 7:70

40. Tai H-C, Schuman EM: Ubiquitin, the proteasome and protein degradation in neuronal function and dysfunction. Nat Rev Neurosci 2008, 9:826-838

41. Shinde VM, Sizova OS, Lin JH, LaVail MM, Gorbatyuk MS: ER stress in retinal degeneration in S334ter Rho rats. PLoS One 2012, 7 : e33266

42. Lin JH, Walter P, Yen TSB: Endoplasmic reticulum stress in disease pathogenesis. Annu Rev Pathol 2008, 3:399-425

43. Choudhury S, Bhootada Y, Gorbatyuk O, Gorbatyuk M: Caspase-7 ablation modulates UPR, reprograms TRAF2-JNK apoptosis and protects T17M rhodopsin mice from severe retinal degeneration. Cell Death Dis 2013, 4:e528

44. Michalakis S, Geiger H, Haverkamp S, Hofmann F, Gerstner A, Biel M: Impaired opsin targeting and cone photoreceptor migration in the retina of mice lacking the cyclic nucleotidegated channel CNGA3. Invest Ophthalmol Vis Sci 2005, 46: $1516-1524$

45. Coleman JE, Zhang Y, Brown GAJ, Semple-Rowland SL: Cone cell survival and downregulation of GCAP1 protein in the retinas of GC1 knockout mice. Invest Ophthalmol Vis Sci 2004, 45: 3397-3403

46. Baehr W, Karan S, Maeda T, Luo DG, Li S, Bronson JD, Watt CB, Yau KW, Frederick JM, Palczewski K: The function of guanylate cyclase 1 and guanylate cyclase 2 in rod and cone photoreceptors. J Biol Chem 2007, 282:8837-8847

47. Azadi S, Molday LL, Molday RS: RD3, the protein associated with Leber congenital amaurosis type 12 , is required for guanylate cyclase trafficking in photoreceptor cells. Proc Natl Acad Sci U S A 2010, 107:21158-21163

48. Kirschman LT, Kolandaivelu S, Frederick JM, Dang L, Goldberg AF, Baehr W, Ramamurthy V: The Leber congenital amaurosis protein, AIPL1, is needed for the viability and functioning of cone photoreceptor cells. Hum Mol Genet 2010, 19:1076-1087

49. Avasthi P, Watt CB, Williams DS, Le YZ, Li S, Chen CK, Marc RE, Frederick JM, Baehr W: Trafficking of membrane proteins to cone but not rod outer segments is dependent on heterotrimeric kinesin-II. J Neurosci 2009, 29:14287-14298

50. Pugh EN Jr, Lamb TD: Phototransduction in vertebrate rods and cones: molecular mechanisms of amplification, recovery and light adaptation. Edited by Stavenga DG, de Grip WJ, Pugh EN Jr. In Handbook of Biological Physics. Amsterdam: Elsevier Science B.V., 2000. pp. $183-255$

51. Jacobson SG, Cideciyan AV, Ratnakaram R, Heon E, Schwartz SB, Roman AJ, Peden MC, Aleman TS, Boye SL, Sumaroka A, Conlon TJ, Calcedo R, Pang JJ, Erger KE, Olivares MB, Mullins CL, Swider M, Kaushal S, Feuer WJ, Iannaccone A, Fishman GA, Stone EM, Byrne BJ, Hauswirth WW: Gene therapy for leber congenital amaurosis caused by RPE65 mutations: safety and efficacy in 15 children and adults followed up to 3 years. Arch Ophthalmol 2012, 130:9-24 